\title{
Two-magnon scattering and the spin-phonon interaction beyond the adiabatic approximation
}

\author{
Matthew J. Reilly and A. G. Rojo \\ Physics Department, The University of Michigan, Ann Arbor, MI 48109-1120
}

\begin{abstract}
We consider a model of Raman scattering for a two-dimensional $S=$ 1/2 Heisenberg Anti-Ferromagnet which includes a dynamical spin-phonon interaction. We observe a broadening of the line shape due to increased coupling with excited high-energy spin states. Our results are close to a model of random, static exchange interactions, first introduced in this context by Haas et al. [J. Appl. Phys. 75, 6340, (1994)], which, when extended to large numbers of spins, explains experiments in the parent insulating compounds of high- $T_{c}$ superconductors.
\end{abstract}

\section{INTRODUCTION}

Since the discovery of high $-T_{c}$ superconductors 1 , the $S=1 / 2$ quantum Heisenberg model has received considerable attention. This is largely due to well-accepted experimental evidence that suggests that these compounds can be described by a two-dimensional (2D) doped Heisenberg model for spin $S=1 / 2$.

One of the experimental techniques used to study the excitations of these systems is Raman scattering. This technique was intensely used in the past for different antiferromagnets of spin $S \geq 1$. For such systems, the main features of the line shape were explained by ParkinsonB, who used a spin-wave theory to account for the photon-two-magnon process 
involved in the Raman scattering. Very good agreement 4 was found for $\mathrm{K}_{2} \mathrm{NiF}_{4}$, which is well described by a spin $S=1$ Heisenberg antiferromagnet.

The results of Raman experiments in the parent insulating compounds of high- $T_{c}$ superconductors, $\mathrm{La}_{2} \mathrm{CuO}_{4}$ and $\mathrm{YBa}_{2} \mathrm{Cu}_{3} \mathrm{O}_{6.2}$, show some qualitative differences with the line shape of previously studied antiferromagnets 0 - As in the case of $\mathrm{K}_{2} \mathrm{NiF}_{4}$, the line shape is centered at an energy corresponding to a spin-pair excitation. However, in contrast with the case of spin $S=1$, the linewidth is very broad, and the spectrum has a very long tail that extends beyond the energy of four magnon excitations. Moreover, while the dominant contribution to scattering is in the so-called $B_{1 g}$ channel, there is also a significant contribution in the nominally forbidden $A_{1 g}$ configurationt.

Since for lower spins the quantum fluctuations are larger, some theoretical analysis beyond the mean-field spin-wave approximation has been attempted to explain the broad line shape. Numerical diagonalizations in a $4 \times 4$ lattice shows very little structure for the $B_{1 g}$ channel: essentially three peaks; a dominant two-magnon peak and two peaks identifiable as four-magnon excitations 8 . This calculation gives vanishing line shape for the $A_{1 g}$ channel. Although the structure of the line shape is clearly different from the one observed in the experiment, the first three moments of both lines are in good agreement. The first three moments of the distribution have been calculated in good agreement to experiment by Singh et al. using cumulant expansions on a Heisenberg model with diagonal next-nearest-neighbor coupling, 9 . Canali and Girvin 10 used the Dyson-Maleev transformation taking into account processes of up to four magnons, and presented convincing evidence that the line shape cannot be explained by quantum fluctuations alone. Raman scattering Hamiltonians with a four-site exchange 11 term have been proposed, these increase the relative weight of the four-magnon scattering, but does not explain the broadening of the peas. This is also consistent with the work of Sugai 12 , and Roger and Delrieu 13. Also, recent studies of spin-pair excitations in a spin $S=1$ system, $\mathrm{NiPS}_{3}$, show a similar linewidth as those observed in the cupratest14.

From the above considerations, one concludes that it is necessary to invoke an additional 
process. It was emphasized by Nori et al. 1 that the spin-phonon interaction can be responsible for the broad linewidth, and for the finite cross-section in the otherwise absent $A_{1 g}$ channel. They supported their arguments by computing the Raman cross section in finite lattices, using a Heisenberg model with random static exchange integrals, and averaging over configurations with equal weight. This calculation is in the spirit of the adiabatic or Born-Oppenheimer approximation 16 , that neglects the fluctuations of the phonon field.

Adding phonons to the 2D $S=1 / 2$ Heisenberg Hamiltonian (HAF) provides a mechanism 15 for the otherwise forbidden $A_{1 g}$ scattering, as well as allowing a coupling between the ground and excited spin states. In a typical HAF, the phonon frequency 5 is about a third of the interaction constant $J$. The first excited state lies at $3 J$, so there is an order of magnitude energy difference between spin and phonon excitations. It was argued that the separation of energy scales justifies the adiabatic phonon approximation.

In the present work we consider the Raman scattering for a Heisenberg model with spinphonon interaction, including the effects of the phonon dynamics. We solve for the exact ground state of a system in which the vibrational degrees of freedom are allowed a finite number of excitations, an approximation valid for small but finite phonon frequencies. To the best of our knowledge, this is the first calculation of exact diagonalizations for a dynamic spin-phonon system. Some work including phonon dynamics beyond the adiabatic approximation exists in the context of one-dimensional Peierls systems. Fradkin and Hirsh 17 studied the electron-phonon interaction using quantum Monte Carlo simulations, and Proetto and Falicov 18 solved the case of two sites and one phonon.

This paper is organized as follows. In Sec. II we present our Hamiltonian and scattering operator and discuss the rationale for our truncated-phonon model. In Sec. III we present computational results and provide theoretical support for these results. We also present an alternative approach to including phonons and compare it to our model. Sec. IV is devoted to conclusions. 


\section{MODEL AND PROCEDURE}

We study a Heisenberg model with spin-phonon interaction described by the following Hamiltonian:

$$
H=\sum_{<i j>}\left\{\left[J-\alpha\left(a_{i j}^{\dagger}+a_{i j}\right)\right] \vec{S}_{i} \cdot \vec{S}_{j}+\omega_{0} a_{i j}^{\dagger} a_{i j}\right\}
$$

where $\vec{S}_{i}$ are spin $1 / 2$ operators, $a_{i j}^{\dagger}\left(a_{i j}\right)$ is a creation (annihilation) operator for an Einstein phonon of frequency $\omega_{0}$, and the simbol " $i j$ " refers to a link of a square lattice. In $J_{i j}=$ $J-\delta J_{i j}$ we include the coupling between the phonons and the spin degrees of freedom through a "displacement" operator $\hat{x}_{i j}=\sqrt{\frac{\hbar}{2 m \omega_{0}}}\left(a_{i j}^{\dagger}+a_{i j}\right)$. Our parameter $\alpha$ in Eq.(11) is

proportional to the spin-phonon coupling constant $\lambda=\alpha \sqrt{2 m \omega_{0} / \hbar}$ relating the change in the exchange integral $\delta J_{i j}$ with the displacement: $\delta J_{i j}=\lambda \hat{x}_{i j}=\alpha\left(a_{i j}^{\dagger}+a_{i j}\right)$.

Due to computational limitations, we need to restrict ourselves to a small number (6 and 8) of spins. To have these as two dimensional as possible, we placed them in non-periodic ladder type structures as shown in Fig. 11.

We use Einstein phonons to simplify the model, and we consider the highest-energy phonons as they are the closest in energy to the magnon excitations and will thus have the greatest coupling to the magnons. The occupation number of the phonon degrees of freedom at each link is in principle unrestricted; the number of phonons ranges from zero to infinity. This makes the problem intractable from the point of view of exact diagonalizations, since the resulting Hilbert space is infinite. In order to overcome this limitation, we restrict the possible number of phonons at each link by imposing the condition $\left(a^{\dagger}\right)^{n}=0$. Computational resources limit us to using $n=2,3$. This approximation maps the phonon degree of freedom into a two- or three-state system at each link. The truncation of the phononic occupation states implies that the variations of $J_{i j}$ due to quantum fluctuations are bounded. For example, for $n=2$, the maximum displacements for a given link is given by the "coherent" states $\left|\psi_{i j}^{ \pm}\right\rangle=2^{-1 / 2}\left(1 \pm a_{i j}^{\dagger}\right)|0\rangle$, and $\delta J_{i j}=\alpha\left\langle\psi_{i j}^{ \pm}\left|\left(a_{i j}^{\dagger}+a_{i j}\right)\right| \psi_{i j}^{ \pm}\right\rangle= \pm \alpha$. Quantum fluctuations themselves are certainly limited by our truncation, but since they are in general small for 
small $\omega_{0}$, our approximation will account for the relevant dynamics in the regime $\delta J_{i j} / J<1$ and $\omega_{0} / J<1$.

We are interested in the Raman scattering intensity $I_{R}(\omega)$, given by:

$$
I_{R}(\omega)=\sum_{\nu}|\langle\nu|\hat{R}| 0\rangle|^{2} \delta\left(\omega-E_{\nu}+E_{o}\right)
$$

where $|\nu\rangle$ are the eigenstates and $E_{\nu}$ the eigenvalues of $H$. We compute $I_{R}(\omega)$ by using the partial fraction expansion method of Gagliano and Balseird19. The relevant operator $\hat{R}$ in

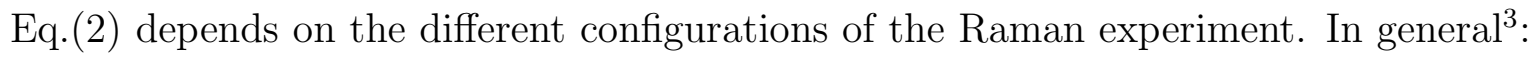

$$
\hat{R}=\sum_{<i j>}\left(\vec{E}_{\text {inc }} \cdot \hat{x}_{i j}\right)\left(\vec{E}_{\mathrm{scat}} \cdot \hat{x}_{i j}\right) \vec{S}_{i} \cdot \vec{S}_{j}
$$

with $\vec{E}_{\text {inc }}$ and $\vec{E}_{\text {scat }}$ refer to the polarization of the incoming and scattered photon respectively, and $\hat{x}_{i j}$ are unit vectors in the near-neighbor directions of the lattice. For square lattices, two common scattering configurations are the so-called $B_{1 g}$ and $A_{1 g}$ symmetries. We let the lattice axis lie along the $x$ and $y$ directions, and we define $x^{\prime}$ and $y^{\prime}$ the directions along the lattice diagonals $\left(\hat{x^{\prime}}=\frac{\hat{y}+\hat{x}}{\sqrt{2}}\right.$ and $\left.\hat{y}^{\prime}=\frac{\hat{y}-\hat{x}}{\sqrt{2}}\right)$, then $B_{1 g}$ corresponds to $\vec{E}_{\text {scat }} \| \hat{x}^{\prime}$ and $\vec{E}_{\text {inc }} \| \hat{y^{\prime}}$, whereas $A_{1 g}$ corresponds to both the incident and scattered photon polarized in the same direction: $\vec{E}_{\text {inc }}\left\|\vec{E}_{\text {scat }}\right\| \hat{x}^{\prime}$.

For the case of the "pure" Heisenberg Hamiltonian (no phonons, or $\alpha=\omega_{0}=0$ ), the absorption corresponding to the $A_{1 g}$ symmetry is zero at any non-zero frequency, since the $A_{1 g}$ operator is proportional to the Hamiltonian. We show in the next section how the inclusion of the phonon dynamics gives rise to a finite absorption in this channel, and argue that a spin-phonon interaction accounts for the main features observed in the Raman experiments in the undoped copper oxides. Also, we compare the results obtained in the dynamical model with that of the Heisenberg model with random (static) exchange integrals, which was studied in this context by Nori et al.15 


\section{RESULTS AND DISCUSSION}

In this section we present numerical results for systems of 6 and 8 spins 20 corresponding to the geometry shown in Figure 1. We first discuss the $A_{1 g}$ symmetry. In this case, and in the absence of lattice distortions, the Raman operator commutes with the Heisenberg Hamiltonian. No line shape is observed in this case: $I_{R}(\omega) \sim \delta(\omega)$. It was first pointed out by Nori et al. 15 that the presence of static disorder in the exchange integrals $J_{i j}$ changes the value of the commutator, and can give rise to a finite $A_{1 g}$ signal quite similar to experiments.

It was argued by Nori et al. 1 罒 that the spin-phonon coupling produces disorder in the values of $J_{i j}$, in the limit where the vibrational motions are much slower than the spin degrees of freedom. Here, we are interested in how this limit is achieved in a system that - up to boundary effects - is translationally invariant, and includes the dynamics of the spin-phonon coupling. Consider our Hamiltonian Eq.(1) and the operator $\hat{R}$ for the $A_{1 g}$ symmetry. For $\omega_{0} \neq 0,[H, \hat{R}] \neq 0$ and we expect a finite line shape. In Fig. 2 we show the $I(\omega)$ obtained for $\alpha=0.1 J$ and $\omega_{0}=0.05 J$. Some of the qualitative features of the experimental line shape are already present in these finite-size systems: there is a broad line shape of width $\sim 2 J$ with a maximum at the two-magnon energy. Note that for our ladder geometries, since most sites have coordination number 3, the two-magnon energy in the Ising limit is located at $2 J$, instead of the corresponding $3 J$ of the square lattice. Due to the finite size of the lattice, the line shape consists of a series of peaks that are centered at the unperturbed energies, which are indicated by arrows in Fig. 2. These unperturbed energies correspond to the manifold of two-and multi-magnon states. In turn, these "internal" peaks have a finite width that is due to the coupling with the phonons. The energy scale dominating this width is $\alpha$.

In order to test the validity of the Hilbert space truncation, we have increased the number of allowed excitations per phonon by one, with no qualitative changes in the line shape (see Fig. 3)

It is interesting to consider the following two limiting cases: $(a)$ vanishing spin-phonon 
coupling $\alpha \rightarrow 0$ for finite phonon frequency $\omega_{0}$, and $(b)$ vanishingi phonon frequency $\omega_{0} \rightarrow 0$ for finite $\alpha$. In both limits the $A_{1 g}$ line shape vanishes. In $(a)$, the spins are uncoupled from the phonons, and the line shape vanishes because in this limit $[H, \hat{R}]=0$. Figure 4 illustrates how the line shape vanishes and shifts to lower energies as $\alpha \rightarrow 0$. In limit $(b)$, the phonons are "static", but remain coupled to the spins, because $\alpha \neq 0$. Since the phonons have no dynamics, the eigenstates can be written in the form

$$
\left|\psi_{\nu}\left(\omega_{0}=0\right)\right\rangle=\left|\psi_{\nu}\{n\}\right\rangle \otimes|\{n\}\rangle,
$$

where $|\{n\}\rangle$ is a static configuration of displacements, in such a way that, for each link $i j$, one has $\left(a_{i j}^{\dagger}+a_{i j}\right)|\{n\}\rangle=\eta_{i j}(\{n\})|\{n\}\rangle$, with $\eta_{i j}(\{n\})$ a $c$-number. The state $\left|\psi_{\nu}\{n\}\right\rangle$ involves spin degrees of freedom only, and is an eigenstate of the following Hamiltonian

$$
H\{n\}=\sum_{<i j>}\left[J-\alpha \eta_{i j}(\{n\})\right] \vec{S}_{i} \cdot \vec{S}_{j} .
$$

For $\omega_{0}=0$, the problem is that of an annealed configuration of displacements: for each configuration $\{n\}$, the couplings $J_{i j}$ are different, and one has to solve for the spin dynamics in the presence of this distribution of couplings. The complete spectrum is obtained by solving $N_{p}$ decoupled eigenvalue problems, with $N_{p}$ being the phononic Hilbert space dimension. For our case of one excitation per link, $N_{p}=2^{N_{\text {links }}}$, with $N_{\text {links }}$ being the number of links of the lattice. What distinguishes this from a quenched disorder is the fact that the ground state of this problem corresponds to the lowest energy state of the spin system in an ordered background of couplings. Since this is also an eigenstate of $\hat{R}$ for the $A_{1 g}$ symmetry, the line shape will be zero. In Fig. 5 we show how the line shape vanishes as $\omega_{0}$ decreases to zero. Note that from this analysis one concludes that, even in the case of $\omega_{0}=0$, the line shape is non-zero at finite temperature. This is due to the presence of thermally excited states, which are eigenstates of a Hamiltonian that does not commute with $\hat{R}$. The right column of Figure 5 shows the rescaled results, which illustrate the line shape approaching a limiting function of $\omega$ whose overall amplitude vanishes as $\omega_{0} \rightarrow 0$. The main features of this limiting function should be accounted for by a model of static disordered couplings. In previous work 
in the context of one-dimensional Peierls systems21, it is argued that in the limit of small phonon frequencies, the quantum lattice fluctuations can be modeled by a static, random Gaussian potential with zero mean. A model in which the exchange integrals are taken from a random configuration of couplings was also studied by Nori et a 15 . We can test this hypothesis in our dynamical model. Results of the comparison between the dynamical and a static disordered system are shown in Fig. 6. Note that both the amplitude and the position of the peaks are in very good mutual agreement. The scattering peaks from the two models have their energies scaled with respect to each other. After they are rescaled, the location and magnitude of the peaks are very close for both models (see Fig. 6). The basis for this rescaling of the horizontal axis is the following. For our dynamic phonon model, the minimum energy state corresponds to the lattice "maximally compressed": $\eta_{i j}=-\langle x\rangle_{0}$. The tendency of the system to compress has the effect of renormalizing the interaction coefficient $J$, since the displacement $x_{i j}$ will tend to be a constant, and therefore $J^{\prime}=J+\alpha\langle x\rangle_{0}$. The average displacement will be greater as the possible number of excitations increases; this explains a shift in energy between 1 and 2 excitations as well.

In the model with static disorder, the phonon energy goes to zero, but the fluctuations are still present. In the treatment of Nori et al.15 the argument used is that the phonon energy is small compared to that of the spin excitations, and thus can be neglected. However, we have shown that for $\omega_{0}$ strictly zero the line shape vanishes. At zero temperature, the disordered model should be compared with the dynamical model at infinitesimal $\omega_{0}$. We prove this statement by using perturbation theory in $\omega_{0}$. Assume an ordering of the states for $\alpha=\omega_{0}=0$, and let us label those states by the index $\nu$. If $\alpha \ll J$ and $\omega_{0}=0$, each state $\left|\psi_{\nu}\right\rangle$ splits in a manifold $\left|\psi_{\nu}\{n\}\right\rangle$ of almost degenerate states of energies $E_{\nu}\{n\}$, that correspond to the "disordered" configurations. If we now turn on $\omega_{0}$, different states are going to mix, in such a way that the ground state can be written as

$$
\left|\psi_{0}\right\rangle \simeq\left|\psi_{0}\{n\}_{0}\right\rangle+\omega_{0} \sum_{\{n\} \neq\{n\}_{0}} c(\{n\})\left|\psi_{0}\{n\}\right\rangle \otimes|\{n\}\rangle,
$$

where $\{n\}_{0}$ is the ordered configuration of couplings. A similar expansion can be used for 
the excited states. If we use the fact that $A_{1 g} \hat{R}$ acts only on the spin degrees of freedom, and that different states $|\{n\}\rangle$ are orthogonal, to lowest order in $\omega_{0}, I(\omega)$ will be given by

$$
I(\omega) \simeq \omega_{0}^{2} \sum_{\{n\}}|c(\{n\})|^{2} \sum_{\nu}\left|\left\langle\psi_{\nu}\{n\}|\hat{R}| \psi_{0}\{n\}\right\rangle\right|^{2} \delta\left(\omega-E_{\nu}\{n\}+E_{0}\{n\}\right) .
$$

We have found computationally a falloff of $I(\omega)$ proportional to $\omega_{0}^{2}$.

Now, if we keep the assumption of $\alpha \ll J$, the states corresponding to each manifold are almost degenerate and we can approximate $c(\{n\}) \sim(M-1)^{-1 / 2} \sim M^{-1 / 2}$, with $M$ the number of configurations $\{n\}$. Therefore, in this limit $I(\omega)$ is given by an average over configurations with equal weight.

We now present results for the $B_{1 g}$ configuration. In Fig. 7 we compare the $B_{1 g}$ scattering from eight $S=1 / 2$ Heisenberg spins for three different cases: bare HAF, with phonon interactions, and with static disorder.

We have argued that peaks at higher excitations will eventually merge into one broad peak as the lattice size increases. To show that this is the case, we have calculated the $A_{1 g}$ and $B_{1 g}$ peaks for a $3 \times 4$ non-periodic lattice with static disorder. The results are shown in Fig. 8. These are in good qualitative agreement with experiments, except that the experimental scattering is centered at $\omega \sim 3 J$ instead of $2 J$ in our case. This is due to finite size effects and to the lattice being non-periodic.

The model with static disorder, when extended to larger numbers of spins than can be obtained with the dynamic phonon model, begins to duplicate experiments. The agreement between the static and dynamic phonon models suggests that the dynamic phonon model will also agree with experiments.

\section{CONCLUSIONS}

In this paper we have presented a model of phonon-magnon interaction with a truncated phonon space. We discussed how this model can explain the otherwise forbidden $A_{1 g}$ scat-

tering and showed that it does in fact give rise to $A_{1 g}$ scattering. We discussed how coupling 
to spin excitations leads to a broadening of the line shape. We have proven that phonons can be modeled by static disorder and compared the results obtained by using quenched phonons to our dynamical model. We showed that the amplitude of the $A_{1 g}$ scattering should fall off as $\omega_{0}^{2}$. We showed that both the $A_{1 g}$ and $B_{1 g}$ scattering is broadened. In conclusion, the broadening is due to coupling to spin excitation states through the phonon interaction.

\section{ACKNOWLEDGEMENTS}

We would like to thank Roberto Merlin and Jim Allen for helpful discussions, and Franco Nori for helpful discussions and a careful reading of this manuscript. 


\section{REFERENCES}

${ }^{1}$ J. G. Bednorz and K. A. Müller, Z. Phys. B 64, 189 (1986).

${ }^{2}$ E. Manousakis, Rev. Mod. Phys. 63, 1 (1991).

${ }^{3}$ J. B. Parkinson, J. Phys. C 2, 2012 (1969).

${ }^{4}$ P. A. Fleury and H. J. Guggenheim, Phys. Rev. Lett. 24, 1346 (1970).

${ }^{5}$ S. Sugai, M. Sato, T. Kobayashi, J. Akimitsu, T. Ito, H. Takagi, S. Uchida, S. Hosoya, T. Kajitani, and T. Fukuda, Phys. Rev. B 42, 1045 (1990).

${ }^{6}$ P. E. Sulewski, P. A. Fleury, K. B. Lyons, S-W. Cheong, and Z. Fisk, Phys. Rev. B 41, 225 (1990).

${ }^{7}$ K. B. Lyons, P. E. Sulewski, P. A. Fleury, H. L. Carter, A. S. Cooper, G. P. Espinosa, Z. Fisk, and S.-W. Cheong, Phys. Rev. B 39, 9693 (1989).

${ }^{8}$ E. Gagliano and S. Bacci, Phys. Rev. B 42, 8772 (1990).

${ }^{9}$ R. R. P. Singh, P. A. Fleury, K. B. Lyons, and P. E. Sulewski, Phys. Rev. Lett. 62, 2736 (1989).

${ }^{10}$ C. M. Canali and S. M. Girvin, Phys. Rev. B 45, 7127 (1992).

${ }^{11}$ Y. Honda, Y. Kuramoto, and T. Watanabe, Physica C 185-189, 1493 (1991).

${ }^{12}$ S. Sugai, Solid State Comm. 75, 795 (1990).

${ }^{13}$ M. Roger and J. M. Delrieu, Synthetic Metals 29, F673 (1989).

${ }^{14}$ S. Rosenblum, A. H. Francis, and R. Merlin, Phys. Rev. B 49, 4352 (1994).

${ }^{15}$ F. Nori, R. Merlin, S. Haas, A. W. Sandvik, and E. Dagotto, Phys. Rev. Lett., 75, 553 (1995); S. Haas, E. Dagotto, J. Riera, R. Merlin, and F. Nori, J. Appl. Phys. 75, 6340 (1994). 
${ }^{16}$ See for example, J. M. Ziman, Electrons and Phonons (Oxford University Press, Oxford, 1960), p. 175.

${ }^{17}$ E. Fradkin and J. E. Hirsch, Phys. Rev. B 27, 1680 (1983).

${ }^{18}$ C. R. Proetto and L. M. Falicov, Phys. Rev. B 39, 7545 (1989).

${ }^{19}$ E. R. Gagliano and C. A. Balseiro, Phys. Rev. Lett. 59, 2999 (1987).

20 The Hilbert space dimension is $\left(\begin{array}{c}N \\ N / 2\end{array}\right) n_{e}^{n_{b}}$, where $N$ is the number of spins, $n_{e}$ is the number of allowed phonon states, and $n_{b}$ is the number of nearest-neighbor pairs. For 6 spins and 2 (3) phonon states in a ladder configuration, this gives a Hilbert dimension of 2560 (43740). For 8 spins, 2 (3) excitations, the dimension becomes 71680 (4133430).

${ }^{21}$ K. Kim, R. H. McKenzie and J. W. Wilkins, Phys. Rev. Lett. 71, 4015 (1993); R. H. McKenzie and J. W. Wilkins, ibid 69, 1085 (1992); S. A. Brazovskii and I. E. Dzyaloshinskii, Zh. Eksp. Teor. Fiz. 71, 2338 (1976) [Sov. Phys. JETP 44, 1233 (1976)]. 


\section{FIGURES}

FIG. 1. Ladder geometries used in our numerical computations. Each dot represents a spin 1/2. Each solid line connects nearest-neighboring spins, and represents a Heisenberg Anti-Ferromagnetic coupling modulated by an Einstein phonon. There is one phonon per coupling.

FIG. 2. Raman cross section $I(\omega)$ versus $\omega / J$ for $A_{1 g}$ scattering. The parameters of the dynamical Hamiltonian are $\alpha=0.1 J, \omega_{0}=0.05 J$, and the number of phonon excitations per link, $n$, is 2 . The vertical scale is arbitrary. The arrows are located at the eigenenergies of the model without phonons corresponding to eigenstates of total $\operatorname{spin} S=0$.

FIG. 3. Comparison between one-and two-phonon (lighter curve) excitations per link, for the Raman line shape $I(\omega)$ in the $A_{1 g}$ symmetry. The parameters are $\alpha=0.1 J$, and $\omega_{0}=0.05 J$, for a ladder of 6 spins.

FIG. 4. Raman line shape for the $A_{1 g}$ symmetry. Here we consider 6 spins (including 1 excitation per link), and $\omega_{0}=0.05 \mathrm{~J}$. The strength of the spin-phonon coupling is varied: (a) $\alpha=0.2 J$, (b) $\alpha=0.1 J$ (c) $\alpha=0.05 J$, (d) $\alpha=0.025 J$. The vertical scale is arbitrary but is the same for the different values of $\alpha$.

FIG. 5. Raman spectrum versus $\omega / J$ for the $A_{1 g}$ symmetry. These results correspond to 6 spins coupled to one phonon excitation and $\alpha=0.1 J$. (a)-(c) are all displayed on the same scale, (d)-(f) have been rescaled. $\omega_{0}=0.3$ for (a) and (d), $\omega_{0}=0.1$ for (b) and (e), and $\omega_{0}=0.025$ for (c) and (f).

FIG. 6. Raman spectra for the $A_{1 g}$ symmetry for 8 spins coupled to one phonon excitation and also scattering from an average over configurations of disorder. (a) $\alpha=0.1 J, \omega_{0}=0.05 J$, and disorder chosen from a Gaussian with variance $\sigma=0.2 \mathrm{~J}$. (b) $\alpha=0.3 \mathrm{~J}, \omega_{0}=0.3 \mathrm{~J}$, and disorder chosen from a Gaussian with variance $\sigma=0.5 \mathrm{~J}$. 
FIG. 7. Raman scattering for the $B_{1 g}$ symmetry using (a) the bare Heisenberg Hamiltonian ( $\alpha=0$ ), (b) with dynamical phonons $\alpha=0.1 J, \omega_{0}=0.05 J$, (c) with dynamical phonons $\alpha=0.3 J$, $\omega_{0}=0.3 J$. The two bottom curves correspond to static Gaussian disorder of variance $\sigma=0.2 J$ $(\mathrm{d})$, and $\sigma=0.5 J(\mathrm{e})$.

FIG. 8. Raman scattering from a $3 \times 4$ non-periodic lattice in the $A_{1 g}$ and $B_{1 g}$ geometries. The spin interactions $J_{i j}$ have static Gaussian disorder of variance $\sigma=0.5$ 